\title{
ASOCIACIÓN ENTRE EL ESTADO MENTAL Y LA CONDICIÓN ORAL DE LA PERSONA ADULTA MAYOR INSTITUCIONALIZADA DE BUCARAMANGA Y SU ÁREA METROPOLITANA: ESTUDIO PRELIMINAR
}

\author{
${ }^{1}$ Genny Patricia Espíndola M., ${ }^{1}$ Ángela Rocío Pinzón C., ${ }^{1}$ Cilia Rosa Daza, ${ }^{2}$ Sonia Constanza Concha S. \\ ${ }^{3}$ Diana Marina Camargo L. \\ ${ }^{1}$ Odontólogas U. Santo Tomas, ${ }^{2}$ Odontóloga, Especialista en Comunicación para la Salud y el Bienestar, \\ Grupo SIBAM, Docente U. Santo Tomás ${ }^{3}$ M.Sc. Epidemiología, Docente, Grupo SIBAM, U. Santo Tomás, Prof. \\ Auxiliar E. Fisioterapia, UIS
}

Autor responsable de correspondencia: Dra. Diana Marina Camargo L.

email:diana_nico@ustabuca.edu.co

RESUMEN

Objetivo: Evaluar la asociación entre algunos factores sociodemográficos y el estado mental con la condición oral de la persona adulta mayor institucionalizada, de Bucaramanga y su área metropolitana.

Materiales y Métodos: Se evaluaron 284 individuos institucionalizados; 45\% fueron mujeres y 55\% hombres, con un promedio de edad de $77.7 \pm 8.8$ años y un rango entre 60 y 96 años.

La función cognitiva se determinó con el Mini-Mental y la Escala de Depresión Geriátrica valoró el nivel de depresión. La condición oral se evaluó por otro grupo de investigación perteneciente a la Universidad Santo Tomás, que trabajó simultáneamente en la recolección de los datos.

Resultados: $\mathrm{El}$ COP-D $\geq 23$, Indice de Caries Radicular (RCI) $\geq 80$, CPITN $\geq 2$ y el edentulismo total fueron los más frecuentes en esta población, con prevalencias de $88.4 \%, 55.2 \%, 84.8 \%$ y 55\%, respectivamente. Se encontró un deterioro mental de 38\%, $40.3 \%$ de depresión leve y $15 \%$ de depresión moderada.

El deterioro mental se asoció con una condición periodontal de leve a severa OR 8.96, IC 95\% (0.72-111.7) ( $\mathrm{p}=0.10)$. A pesar de no hallar significancia estadística para los otros indicadores orales, es importante considerar la condición mental como factor de riesgo importante para tener una mala higiene oral ( $\mathrm{ICP} \geq 2$ ), $\mathrm{RCI} \geq 80$ y $\mathrm{CPITN} \geq 2$. La condición mental no se encontró asociada con edentulismo total en este estudio.

Conclusiones: Es necesario valorar integralmente la persona mayor, para determinar como interactúan aspectos sociales, culturales, funcionales, sensoriales y mentales sobre su salud oral y bienestar. [Espíndola G, Pinzón Á, Daza C, Concha S, Camargo D. Asociación entre el estado mental y la condición oral de la persona adulta mayor institucionalizada de Bucaramanga y su área metropolitana: estudio preliminar. Ustasalud 2003;1:21-32]

PAL.ABRAS CLAVES: Nivel cognitivo, Depresión, Escala de depresión geriátrica, MMSE, Morbilidad oral.

Association Between Mental Status and Oral Condition in Institutionalized Elderly People in the City of Bucaramanga and Metropolitan Area: Preliminar Study

\begin{abstract}
Purpose: The purpose of this study was to evaluate the association between some sociodemographic factors and mental status with oral condition in institutionalized elderly people from Bucaramanga and metropolitan area.

Material and Methods: The group of patients studied were 284, the mean age of the patients was $77.7 \pm 8.8 \mathrm{yr}, 45 \%$ were female and 55\% men. Cognitive function was measure with mini-mental and geriatric depression scale established a depression level. Oral condition was evaluated for another research group of Santo Tomas University who worked simultaneously. Results: DMFT Index $\geq 23$, Root Caries Index $\geq 80$, CPITN $\geq 2$ and edentulism were the most prevalent conditions, 88.4\%, 55.2\%, $84.8 \%$ and $55 \%$ respectively. We found a mental impaired of $38 \%$, mild depression $40.3 \%$ and moderate depression $15 \%$. Mental impairment was associated with a periodontal condition from mild to severe OR 8.96, IC 95\% (0.72-111.7) $(\mathrm{P}=0.10)$.

Conclusions: Even no significance statistic was found to oral indicator, is important to consider mental condition like a potential risk factor to poor oral hygiene (Plaque Index $\geq 2$ ), $R C I \geq 80$ and CPITN $\geq 2$. Besides that, the mental condition was no associated with total edentulism in this study, is necessary to value elderly person to determine how social and culture factors, functional skills, sensorial faculties and mental condition operate on oral health and welfare.
\end{abstract}

KEY WORDS: Mental status, Depression, Geriatric depression scale, MMSE, Oral condition. 


\section{INTRODUCCIÓN}

La especie humana está envejeciendo. La sociedad, en general es más vieja que nunca antes en la historia de la humanidad. La esperanza media de vida en el momento del nacimiento ha aumentado 20 años desde 1950, hasta los 66 años en la actualidad, y se espera que a mediados del siglo aumente 10 años más. Por lo que se proyecta que las personas mayores pasen de 600 millones en el año 2000 a 2000 millones en el año 2050. ${ }^{1}$

En los países en desarrollo el envejecimiento es un tema importante. Las proyecciones indican que envejecerán rápidamente en la primera mitad del siglo XXI y se espera que la proporción de personas mayores se incremente de 8 a 19\%, en 2050, mientras que los niños disminuirán de 33 a $22 \%{ }^{1}$

Entre los compromisos y programas centrales de Naciones Unidas se encuentran: Oferta de asistencia sanitaria y apoyo a las personas mayores, según sus necesidades, $\mathrm{y}$ además, aprovechar la investigación científica y los conocimientos de expertos para entrenarse en las implicaciones individuales, sociales y sanitarias del envejecimiento, especialmente en países en vía de desarrollo, como Colombia. ${ }^{1}$

Nuestro país ha sido catalogado como de envejecimiento intermedio. La disminución de la fecundidad, la mortalidad infantil y el aumento de la esperanza de vida a 69.3 años incrementará la proporción de personas ancianas de $6.7 \%$, en 1998 , a $14.8 \%$ en $2025 .{ }^{2}$

La salud integral y el bienestar de la persona mayor compete a todos los estamentos de la Nación. Los profesionales de la salud debemos prepararnos para enfrentar con calidad, pertinencia e idoneidad, el reto que representa el cambio demográfico que se proyecta.

Según el Estudio Nacional de Enfermedad Bucodental (ENSAB III), se ha observado una lenta pero progresiva recuperación de la salud bucal en los últimos años. A pesar de lo anterior, el deterioro de la condición bucodental se produce rápidamente con el paso de la adolescencia a la edad adulta, dado que el porcentaje de dientes sanos se reduce al $50 \%{ }^{3}$

El COP-D es de 19.7\% destacándose un aumento del 100\% en el número de dientes perdidos por caries. Así mismo se observa la necesidad de tratamiento periodontal, ya que el 96\% de esta población presenta sangrado y cálculos distribuidos, por lo menos en la mitad de la boca, las bolsas, poco o muy profundas, se encuentran por lo menos en un sextante. ${ }^{3}$

Entre los aspectos importantes por considerar en las personas mayores están la depresión y la función cognitiva. Se estima que en los países europeos entre un 10 y $15 \%$ de personas sobre los 65 años presentan algún déficit cognitivo y $5 \%$ demencia. La prevalencia de síntomas depresivos es aproximadamente de $15 \%{ }^{4}$

El estado mental de la persona mayor se refiere no sólo a procesos mentales superiores, sino a aspectos emocionales, funcionales, nutricionales y afectivos, implicados en la vida cotidiana. ${ }^{2,5}$ Conocer cómo operan los procesos de conocimiento en la vejez y cuáles son las alteraciones mentales prevalentes en esta importante fase de la vida, las tendencias de los indicadores y factores que influencian la salud en general y la salud oral, en especial, deben conducir a diseñar e implementar programas de intervención, cuyo objetivo prioritario sea contribuir a mejorar la calidad de vida de las personas mayores. ${ }^{6}$

Como una aproximación inicial al conocimiento de la situación de salud en este grupo poblacional en nuestra región y en el país, se realizó este estudio con el propósito de evaluar la asociación entre algunos factores sociodemográficos y el estado mental, con la condición oral de la persona adulta mayor institucionalizada, de Bucaramanga, y su área metropolitana.

\section{MATERIALES Y MÉTODOS}

Se realizó un estudio de corte transversal, ${ }^{7}$ cuya población de estudio fueron 1.117 personas mayores de 60 años, de ambos géneros, residentes en hogares, asilos 0 ancianatos de Bucaramanga y su área metropolitana, entre marzo y abril de 2002.

Cada institución fue notificada con anterioridad por medio de una carta en la que se explicaba el motivo del estudio y se solicitaba la autorización.

La muestra fue calculada mediante la rutina sample-size para estudios de corte transversal, del programa EPI-INFO v.6.04d $\mathrm{d}^{8}$ con base en las siguientes especificaciones de diseño: prevalencia de enfermedades bucodentales en el grupo no expuesto 30\%, OR (Odds Ratio) 2.0, confianza 
$95 \%$, poder $80 \%$ y razón $1: 1$; lo que permitió estimar que una muestra aproximada de 300 individuos, detectaría asociaciones estadísticamente significativas. Los individuos fueron seleccionados mediante un muestreo aleatorio simple.

Fueron incluidos en el estudio individuos con antecedentes de demencia, tratamiento médico o farmacológico actual a quienes se les hubiera realizado previamente un examen estomatológico, aplicado por otro grupo de estudiantes de la Universidad Santo Tomás, en el mismo momento.

Como criterios de exclusión se definieron: Individuos sordos o que presentaran retraso mental, incapacidad motriz severa, individuos con problemas de esquizofrenia 0 afasia según la historia clínica.

Se recolectaron variables sociodemográficas generales como edad, género, ocupación laboral dentro de la institución, seguridad social y escolaridad como número de años estudiados y aprobados. Adicionalmente se registró la presencia de antecedentes médicos generales.

El Índice Cuantificado de Placa (ICP) ${ }^{9}$ se calificó como bueno para rangos entre 0 y 0.9 , regular entre 1 y 1.9 y malo entre 2 y 3.

El estado de la dentición y las necesidades de tratamiento se determinaron aplicando el índice de cariados, obturados y perdidos COP-D. ${ }^{3}$

También se estableció el índice de caries radicular RCI siguiendo las recomendaciones de Katz, publicadas por Wallace. $^{10}$

El Índice Periodontal Comunitario de Necesidades de Tratamiento, CPITN ${ }^{11}$ se clasificó de la siguiente manera: 0 $=$ Periodonto sano; 1 = Sangrado al sondaje (gingivitis marginal); $2=$ Presencia de cálculos supragingivales (periodontitis incipiente); $3=$ Bolsa de 4 a $5 \mathrm{~mm}$ presencia de cálculo y placa subgingival (periodontitis moderada), y $4=$ Bolsas mayores de $6 \mathrm{~mm}$ (periodontitis avanzada)

El estado protésico se determinó por separado para el maxilar superior e inferior, al igual que la necesidad de cambio y la necesidad de elaboración de prótesis. ${ }^{3}$
Finalmente, el edentulismo parcial se registró como presente, si el individuo era edéntulo en alguna de sus hemiarcadas, y total, si el individuo era totalmente edéntulo.

El estado mental o función cognitiva se determinó mediante la aplicación del Examen Mental Abreviado MiniMental(MMSE), estableciendo los puntos de corte para deterioro mental según la escolaridad del individuo. ${ }^{12} \mathrm{El}$ instrumento registra varios atributos como: orientación, retención, atención y cálculo, evocación o recordación, lenguaje y copia.

Si el individuo presenta o evidencia alteración visual se suman dos puntos al puntaje total, ya que esta deficiencia puede proporcionar falsos positivos por una discapacidad orgánica, mas no cognitiva. Respecto de la edad se suma un punto, si es mayor de 65 años, y dos puntos, si es mayor de 75 años. ${ }^{12}$

El nivel de depresión se evaluó mediante la Escala de Depresión Geriátrica. Consta de 15 preguntas breves y concretas, que requieren una respuesta simple y precisa, Si - No. Evalúa el nivel de depresión que maneja el individuo. Las respuestas que sugieren depresión se encuentran en negrilla, mayúsculas y codificadas con el número uno. Un puntaje entre 0 y 4 sugiere normalidad; entre 5 y 9 , depresión leve y de 10 a 15, depresión moderada. ${ }^{12}$

Previa recolección de la información se realizó inicialmente un entrenamiento para el manejo de formularios y posteriormente, se hizo una prueba piloto con 4 adultos mayores voluntarios, con el fin de evaluar la reproducibilidad entre evaluadores, verificar la utilidad del instrumento y estimar tiempos y rendimientos al aplicar el Mini-Mental y la Escala de Depresión Geriátrica.

\section{Análisis}

Se describió la población de estudio en todas sus variables aplicando medidas de tendencia central y dispersión y tablas de frecuencia, según la naturaleza y distribución de las mismas. ${ }^{13}$

El análisis de la asociación entre la condición oral y los factores sociodemográficos, antecedentes médicos, estado mental y nivel de depresión se realizó aplicando un análisis de regresión logística simple, que estimó las razones relativas (OR) y sus intervalos de confianza del $95 \% .{ }^{14}$ Se definieron como variables dependientes en forma dicotómica (1/0) las siguientes: ICP $\geq 2$, COP-D $\geq 23, R C I \geq 20$, 
CPITN $\geq 2$, edentulismo total vs. parcial, ausencia de prótesis, necesidad de cambio y de elaboración de prótesis para cada maxilar, superior e inferior.

Como variables explicatorias se analizaron: género, edad, escolaridad, antecedentes médicos, deterioro mental y nivel de depresión.

Finalmente, se elaboró un modelo múltiple para tratar de explicar cada variable dependiente siguiendo las recomendaciones de Hosmer y Lemeshow, también se evaluó su bondad de ajuste. ${ }^{15}$

El análisis consideró dos niveles de significancia: $\alpha=$ 0.05 y 0.10 . La base de datos se elaboró en Excell ${ }^{16} \mathrm{y}$ el análisis en STATA 6.0. ${ }^{17}$

Todos los procedimientos siguieron las recomendaciones de la Resolución 8430 de 1993, del Ministerio de Salud, para el trabajo con personas mayores. Además se solicitó la participación voluntaria tanto de la institución como de la persona mayor institucionalizada, para ingresar al estudio.

\section{RESULTADOS}

\section{Descripción General}

En total se evaluaron 284 individuos institucionalizados. La distribución por género mostró para mujeres $45 \%$ y para hombres $55 \%$, con un promedio de edad de $77.7 \pm$ 8.8 años y un rango entre 60 y 96 años.

El promedio de escolaridad fue de 2.4 años \pm 3 años, con un rango entre 0 y 18 años. El $42 \%$ de la población es analfabeta, el 48.16\% tuvieron de 1 a 5 años de escolaridad, el 8.37\% cursó de 6 a 11 años y tan sólo el 1\% restante estudió más de 11 años.

Los antecedentes médicos más frecuentes fueron: consumo de medicamentos de control (51.76\%), hipertensión arterial HTA (39\%), nervios (estrés, 38.7\%) y problemas renales $(22 \%)$ (Figura 1$)$.

Los hallazgos de la condición odontológica general se presentan en la Tabla 1, en la que son evidentes los altos indices para todas las variables de estudio.
Tabla 1. Condición oral de los adultos mayores institucionalizados evaluados

\begin{tabular}{|c|c|}
\hline Variable & Pocentaje \\
\hline \multicolumn{2}{|l|}{ ICP $(n-99)$} \\
\hline Bueno & 9.1 \\
\hline Regular & 20.2 \\
\hline Malo & 70.7 \\
\hline \multicolumn{2}{|l|}{ CPITN (n:92) } \\
\hline Periodonto sano & 6.5 \\
\hline Gingivitis marginal & 8.7 \\
\hline Per. leve & 21.7 \\
\hline Per. moderada & 39.1 \\
\hline Per. severa & 23.9 \\
\hline Edentulismo total (n:282) & 55 \\
\hline $\operatorname{COP}(d)(n-284)$ & $27^{+}(8-28)$ \\
\hline $\mathrm{RCl}(\mathrm{n}: 131)$ & $31^{*}(0-50)$ \\
\hline
\end{tabular}

\section{Valoración del estado mental}

\section{Minimental}

Sólo 274 individuos pudieron ser examinados; diez fueron excluidos por problemas cognitivos severos, que no permitieron su valoración.

Al totalizar el MMSE, considerando el nivel de escolaridad de cada individuo y siguiendo las recomendaciones de McDowell, ${ }^{12}$ se estableció un examen mental normal para 170 individuos (62.04\%) y de deterioro cognitivo para 104 (37.96\%) del total de la población evaluada.

\section{Escala de Depresión Geriátrica}

De los 274 individuos evaluados 122 (44.7\%), no estaban deprimidos; 110 (40.3\%) mostraron depresión leve, y 42 (15\%) registraron una depresión moderada.

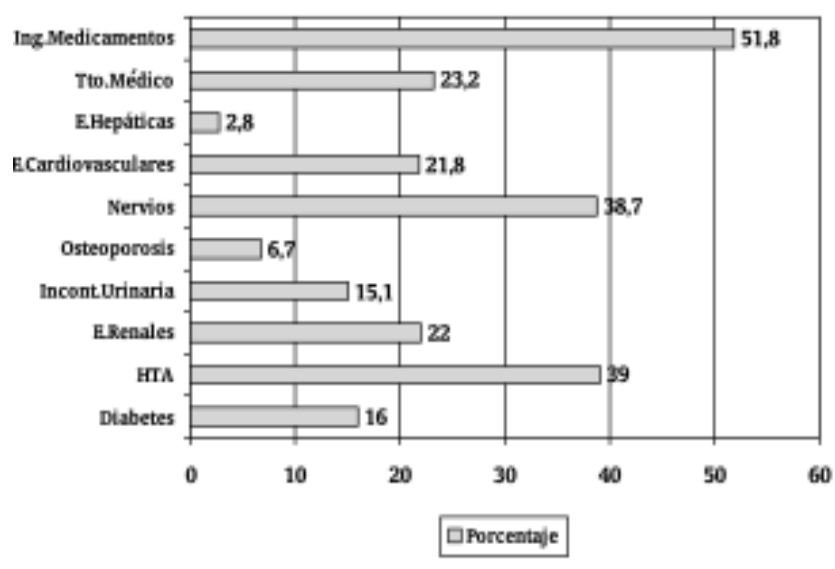

Figura 1. Frecuencias de los antecedentes médicos encontrados ( $\mathrm{n}=$ 284) 


\section{Análisis Bivariado}

Los resultados de regresión logística, tanto simple como múltiple, para ICP, se presentan en la Tabla 2 . El género masculino se presentó como factor de riesgo OR 2.04 y la escolaridad como factor protector OR 0.29 para seis o más años de escolaridad, al igual que los antecedentes médicos en general.

A pesar de no ser estadísticamente significativos, el deterioro mental y el nivel de depresión se comportan como factores de riesgo, que pueden ser importantes, si se con sideran los límites superiores de los intervalos de confianza del 95\%.

El índice COP-D no presentó asociaciones estadísticamente significativas (Tabla 3). Para RCI, los antecedentes médicos, como problemas renales, artritis e ICP regular son claros factores de riesgo con OR de 4.1, 2.54 y 10 , respectivamente. El consumo de medicamentos fue protector $(\mathrm{OR}=0.43)$. Las demás variables no fueron estadísticamente significativas (Tabla 4).

Tabla 2. Asociación entre factores sociodemográficos, antecedentes médicos y condición mental con el Índice Cuantificado de Placa (ICP)

\begin{tabular}{|c|c|c|c|c|}
\hline \multicolumn{5}{|c|}{ ICP } \\
\hline FACTOR & $\begin{array}{c}\geq_{\text {Regular-Malo }} \\
\text { n:70 }\end{array}$ & $\begin{array}{c}1 \\
\text { Bueno } \\
n: 29\end{array}$ & OR & IC $95 \%$ \\
\hline \multicolumn{4}{|l|}{ Edad (años) } & $0.83 \cdot 5.03$ \\
\hline $60-75$ & 32 & 16 & 1.0 & \\
\hline $76-89$ & 35 & 11 & 1.59 & $0.64-3.93$ \\
\hline 90 y más & 3 & 2 & 0.75 & $0.11-4.95$ \\
\hline \multicolumn{5}{|l|}{ Escolaridad (âิos) } \\
\hline 0 & 27 & 9 & 1.0 & \\
\hline 1.5 & 33 & 11 & 1.0 & $0.36=2.76$ \\
\hline $6 \mathrm{y}$ más & 7 & 8 & 0.29 & $0.08 \cdot 1.03$ \\
\hline \multicolumn{5}{|l|}{ Antecedentes médicos } \\
\hline Diabetes & 10 & $\mathrm{~g}$ & 0.44 & $0.15=1.22$ \\
\hline HTA & 24 & 16 & 0.42 & $0.18 \cdot 1.01$ \\
\hline Tratamiento médico & 31 & 21 & 0.30 & $0.12-0.76$ \\
\hline \multicolumn{5}{|l|}{ Depresión } \\
\hline Ausente & 28 & 15 & 1.0 & \\
\hline Leve & 27 & 12 & 1.20 & $0.48 \cdot 3.04$ \\
\hline Moderada & 12 & 1 & 6.42 & $0.76-54.3$ \\
\hline \multicolumn{5}{|l|}{ Modelo Múltiple } \\
\hline Género masculino & & & 3.04 & $1.03 \cdot 9.01$ \\
\hline Escolarided (años) & & & 0.89 & $0.78-1.0$ \\
\hline Tratamiento médico & & & 0.21 & $0.07-0.64$ \\
\hline
\end{tabular}

Tabla 3. Asociación entre factores sociodemográficos, antecedentes médicos y condición mental con el Índice COP-D

\begin{tabular}{|c|c|c|c|c|}
\hline \multirow[b]{2}{*}{ EACTOR } & \multicolumn{2}{|c|}{ COPD } & \multirow[b]{2}{*}{ OR } & \multirow[b]{2}{*}{ IC $95 \%$} \\
\hline & $\underset{\mathrm{B}: 251}{\geq 23}$ & $\begin{array}{l}<23 \\
n: 33\end{array}$ & & \\
\hline $\begin{array}{l}\text { Género masculino } \\
\text { Edad (ahos) }\end{array}$ & 134 & 20 & 0.81 & $0.39-1.67$ \\
\hline $60-75$ & 98 & 14 & 1.0 & \\
\hline $76-59$ & 123 & 14 & 1.26 & $0.57-2.76$ \\
\hline $90 \mathrm{y}$ mass & 21 & 5 & 0.60 & $0.19=1.85$ \\
\hline \multicolumn{5}{|l|}{ Escolaridad (años) } \\
\hline 0 & 96 & 14 & 1.0 & \\
\hline 1.5 & 110 & 13 & 1.23 & $0.55=2.75$ \\
\hline 6 y mas & 21 & 5 & 0.60 & $0.20-1.90$ \\
\hline $\begin{array}{c}\text { Deterioro mental } \\
\text { Depresibin }\end{array}$ & 85 & 12 & 1.0 & $0.47-2.11$ \\
\hline Ausente & 105 & 12 & 1.0 & \\
\hline Leve & 87 & 15 & 0.66 & $0.29=1.48$ \\
\hline $\begin{array}{l}\text { Moderada } \\
\text { ICP }\end{array}$ & 33 & 5 & 0.75 & $0.25-2.28$ \\
\hline Bueno & 7 & 1 & 1.0 & \\
\hline Regular & 18 & 2 & 1.29 & $0.10-165$ \\
\hline
\end{tabular}


Tabla 4. Asociación entre factores sociodemográficos, antecedentes médicos y condición mental con el Índice de Caries Radicular (RCI).

\begin{tabular}{|c|c|c|c|c|}
\hline \multicolumn{5}{|c|}{ RCI } \\
\hline \multirow[t]{2}{*}{ FACTOR } & $\geq 20$ & $<20$ & OR & IC 95\% \\
\hline & n:77 & $n=51$ & & \\
\hline Género masculino & 56 & 21 & 2.02 & $0.96-4.20$ \\
\hline \multicolumn{5}{|l|}{ Edad (años) } \\
\hline $60-75$ & 36 & 18 & 1.0 & \\
\hline $76-89$ & 45 & 21 & 1.07 & $0.50-2.30$ \\
\hline 90 y más & 4 & 4 & 0.5 & $0.10-2.2$ \\
\hline \multicolumn{5}{|l|}{ Escolaridad (años) } \\
\hline 0 & 33 & 15 & 1.0 & \\
\hline 1.5 & 33 & 22 & 0.68 & $0.30-1.53$ \\
\hline 6 y más & 12 & 5 & 1.10 & $0.32-3.65$ \\
\hline \multicolumn{5}{|l|}{ Antecedentes médicos } \\
\hline Problemas Renales & 25 & 4 & 4.10 & $1.32-12.7$ \\
\hline Artritis & 28 & 7 & 2.54 & $1.02-6.30$ \\
\hline Consumo de medicamentos & 51 & 33 & 0.43 & $0.19-1.01$ \\
\hline Deterioro mental & 25 & 16 & 0.77 & $0.35-1.66$ \\
\hline \multicolumn{5}{|l|}{ Depresión } \\
\hline Ausente & 37 & 16 & 1.0 & \\
\hline Leve & 28 & 19 & 0.64 & $0.28-1.45$ \\
\hline Moderada & 13 & 7 & 0.80 & $0.27-2.38$ \\
\hline \multicolumn{5}{|l|}{ ICP } \\
\hline Bueno & 2 & 6 & 1.0 & \\
\hline Regular & 10 & 3 & 10.0 & $1.28-78.1$ \\
\hline Malo & 32 & 16 & 6.0 & $1.09-33.1$ \\
\hline
\end{tabular}

Los hallazgos para Índice Periodontal Comunitario tan sólo muestran OR significativos con antecedentes médicos, los cuales se comportan como factores protectores. En forma similar al COP-D, el ICP es un claro factor de riesgo, OR 24 para ICP malo. (Tabla 5).

Para el CPITN $\geq 2$ el deterioro mental se encontró como factor de riesgo OR 3.78 en un nivel $\alpha=0.10$. El nivel de depresion no fue estadísticamente significativo (Tabla 5).

El análisis de asociación entre los factores de exposición para ser edéntulo total, comparado con el parcial, identificó al género masculino como protector OR 0.57, al igual que estar en tratamiento médico y consumo de medicamentos de control OR 0.56 y 0.59 respectivamente.

El estado mental y el nivel de depresión se encontraron con riesgos cercanos al valor nulo, por lo que no fueron significativos (Tabla 6).

Finalmente, la evaluación de los factores de exposición asociados al estado protésico, se muestra en la Tabla 7, de la cual es importante destacar como factores de riesgo el género masculino, la edad y el deterioro mental para ausencia, necesidad de cambio y elaboración de prótesis en ambos maxilares.

La escolaridad, en general, es un factor protector para cualquiera de las condiciones evaluadas.

\section{Análisis Multivariado}

Para ICP el modelo final fue el siguiente: ICP $\geq 2=1.46+$ 1.11 (género masculino) - 1.55 (tratamiento médico) - 0.12 (escolaridad). Los resultados de los OR ajustados se presentan en la Tabla 2 . El $\chi^{2}$ de bondad de ajuste mostró una $\mathrm{p}=0.44$ con un $\mathrm{n}=95$.

No se encontró un modelo múltiple que explicara la condición oral para COP-D o RCI, al considerar los niveles de significancia definidos.

El Índice Periodontal Comunitario de necesidades de tratamiento se explica con este modelo: $\mathrm{CPITN} \geq 2=2.15+$ 2.19 (deterioro mental) - 2.07(Enfermedad cardiovascular) -2.2 (consumo de medicamentos de control) +3.71 (ICP $\geq 2$ ). Los riesgos ajustados se muestran en la Tabla 5. El $\chi^{2}$ de bondad de ajuste mostró una $\mathrm{p}=0.96$ con $\mathrm{n}=86$. 
Tabla 5. Asociación entre factores sociodemográficos, antecedentes médicos y condición mental con el Índice Periodontal Comunitario (CPITN).

\begin{tabular}{|c|c|c|c|c|}
\hline \multirow[b]{2}{*}{ FACTOR } & \multicolumn{2}{|c|}{ CPIrN } & \multirow[b]{2}{*}{ OR } & \multirow[b]{2}{*}{ IC $95 \%$} \\
\hline & $\underset{\mathrm{n}: 78}{\geq 2}$ & $\begin{array}{c}<2 \\
\mathrm{n}: 14\end{array}$ & & \\
\hline Género masculino & 52 & 8 & 1.50 & $0.49-4.61$ \\
\hline \multicolumn{5}{|l|}{ Edad (anos) } \\
\hline $60-75$ & 38 & 6 & 1.0 & \\
\hline $76-89$ & 37 & 7 & 0.83 & $0.26-2.70$ \\
\hline 90 y más & 3 & 1 & 0,47 & $0.04-5.30$ \\
\hline \multicolumn{5}{|l|}{ Escolaridad (años) } \\
\hline 0 & 30 & 4 & 1.0 & \\
\hline 1.5 & 35 & 8 & 0.58 & $0.16-2.13$ \\
\hline $6 y$ más & 10 & 2 & 0.66 & $0.11-4.20$ \\
\hline \multicolumn{5}{|l|}{ Antecedentes médicos } \\
\hline Diabetes & 11 & 5 & 0.30 & $0.09-0.99$ \\
\hline Enfermedad cardiovascular & 18 & 7 & 0.30 & $0.10-0.93$ \\
\hline Tratamiento médico & 36 & 12 & 0.14 & $0.03-0.68$ \\
\hline Consumo de medicamentos & 53 & 13 & 0.16 & $0.02-1.03$ \\
\hline Deterioro mental & 29 & 2 & 3.78 & $0.79-18.3$ \\
\hline \multicolumn{5}{|l|}{ Depresión } \\
\hline Ausente & 33 & 8 & 1.0 & \\
\hline Leve & 30 & 5 & 1.45 & $0.42-4.93$ \\
\hline Moderada & 12 & 1 & 2.90 & $0.33-25.8$ \\
\hline \multicolumn{5}{|l|}{ ICP } \\
\hline Bueno & 5 & 4 & 1.0 & \\
\hline Regular & 11 & 7 & 1.25 & $0.25-6.30$ \\
\hline Malo & 60 & 2 & 24.0 & $3.49-164.9$ \\
\hline \multicolumn{5}{|l|}{ Modelo Múltiple } \\
\hline Enfermedad cardiovascular & & & 0.13 & $0.018-0.88$ \\
\hline Deterioro mental & & & 8.96 & $0.72-111.7$ \\
\hline Consumo de medicamentos & & & 0.35 & $0.11-1.10$ \\
\hline $\mathrm{ICP} \geq 2$ & & & 40.7 & $5.12-324.6$ \\
\hline
\end{tabular}

Tabla 6. Asociación entre factores sociodemográficos, antecedentes médicos y condición mental con el Edentulismo Total.

\begin{tabular}{|c|c|c|c|c|}
\hline \multirow[b]{2}{*}{ FACTOR } & \multicolumn{2}{|c|}{ Edentulismo } & \multirow[b]{2}{*}{ OR } & \multirow[b]{2}{*}{ IC $95 \%$} \\
\hline & $\begin{array}{l}\text { Total } \\
\text { n:155 }\end{array}$ & $\begin{array}{c}\text { Parcial } \\
\text { n:127 }\end{array}$ & & \\
\hline Género masculino & 75 & 79 & 0.57 & $0.35-0.94$ \\
\hline \multicolumn{5}{|l|}{ Edad (años) } \\
\hline $60-75$ & 54 & 59 & 1.0 & \\
\hline $76-89$ & 87 & 57 & 1.67 & $1.01-2.74$ \\
\hline $90 \mathrm{y}$ más & 14 & 11 & 1.39 & $0.58-3.32$ \\
\hline \multicolumn{5}{|l|}{ Escolaridad (años) } \\
\hline 0 & 63 & 50 & 1.0 & \\
\hline $1-5$ & 75 & 55 & 1.08 & $0.65-3.32$ \\
\hline 6 y más & 10 & 16 & 0.50 & $0.21-1.18$ \\
\hline \multicolumn{5}{|l|}{ Antecedentes médicos } \\
\hline Enfermedades hepáticas & 7 & 1 & 6.0 & $0.73-49.4$ \\
\hline Tratamiento médico & 72 & 73 & 0.64 & $0.40-1.03$ \\
\hline Consumo de medicamentos & 92 & 92 & 0.56 & $0.34-0.92$ \\
\hline Deterioro mental & 55 & 47 & 0.93 & $0.57-1.52$ \\
\hline \multicolumn{5}{|l|}{ Depresión } \\
\hline Ausente & 66 & 53 & 1.0 & \\
\hline Leve & 59 & 50 & 0.95 & $0.56-1.59$ \\
\hline Moderada & 22 & 18 & 0.98 & $0.48-2.02$ \\
\hline \multicolumn{5}{|l|}{ Modelo Múltiple } \\
\hline Género masculino & & & 0.57 & $0.35-0.94$ \\
\hline Edad (años) & & & 1.02 & $1.00-1.06$ \\
\hline Consumo de medicamentos & & & 0.59 & $0.35-0.99$ \\
\hline
\end{tabular}


Tabla 7. Asociación entre factores sociodemográficos y estado mental con el estado protésico.

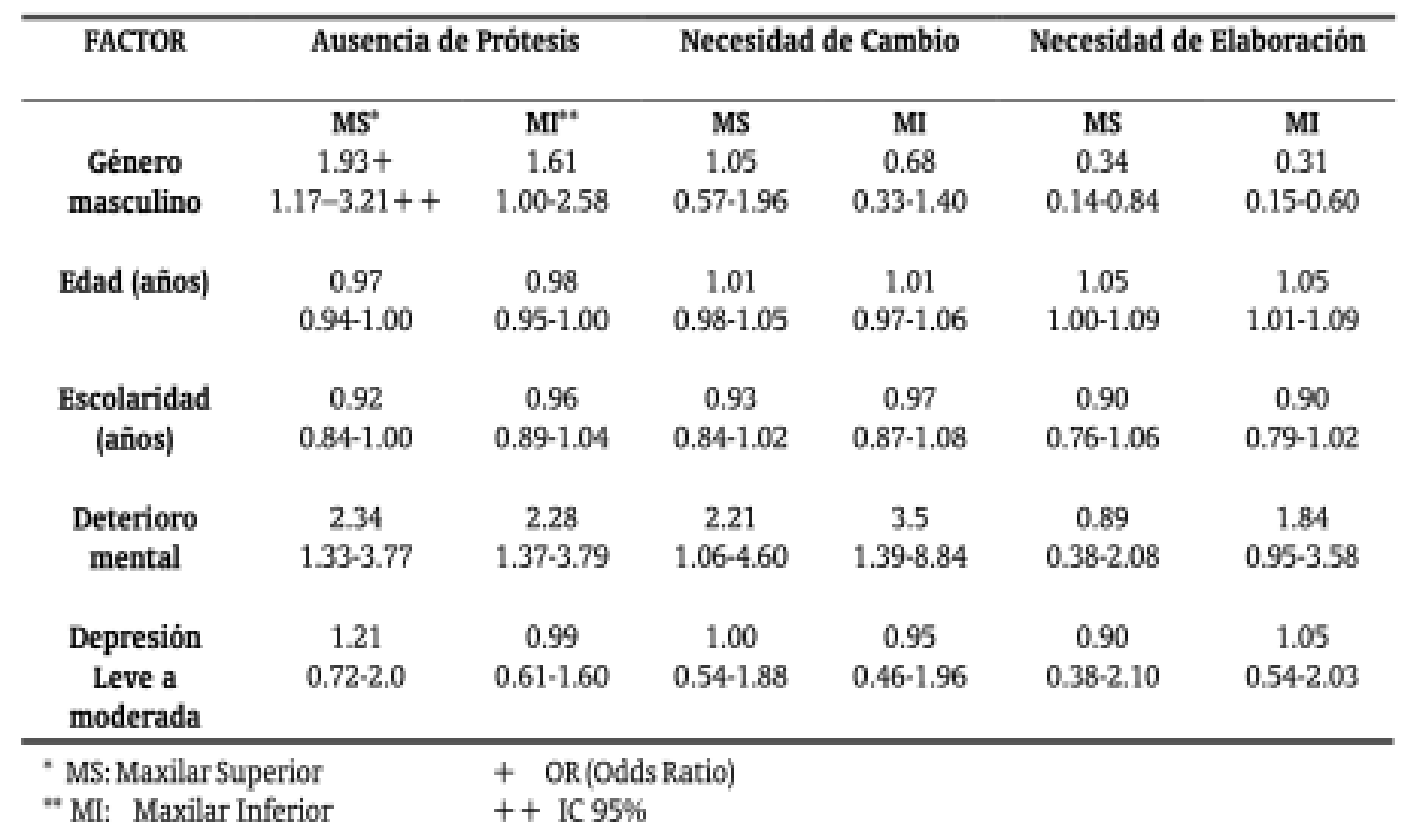

El edentulismo total se explica de la siguiente manera: Edéntulo total $=-1.89-0.53$ (género masculino) +0.036 (edad) - 0.63 (consumo de medicamentos). El $\chi^{2}$ de bondad de ajuste mostró una $\mathrm{p}=0.44$, con $\mathrm{n}=267$ (Tabla 6).

\section{DISCUSIÓN}

Como en muchos otros campos de la geriatría, la odontología se enfrenta a una serie de generalizaciones, acerca de los efectos del envejecimiento sobre la cavidad bucal. Un ejemplo claro es que aunque muchos ancianos tienen buena salud oral, suele admitirse, como parte del proceso normal de envejecimiento, que las personas de edad avanzada pierden los dientes, tienen atrofia de la mucosa bucal, gran frecuencia de caries cervicales, atrofia de la musculatura bucofacial, alteraciones de volumen y composición de la saliva, desgastes de los tejidos duros y disminución en las funciones sensoriales de gusto, olfato y tacto, entre otras. ${ }^{18}$

Estos cambios justifican la necesidad de prestar un servicio odontológico integral para la población geriátrica, ajustado a la percepción que tiene de su cavidad bucal, su salud en general y el impacto o valor en su vida diaria de diferentes aspectos fisiológicos, psicológicos y sociales, con el fin de mejorar las estrategias usadas en el trabajo con este grupo poblacional. ${ }^{19}$

La valoración del estado mental es de vital importancia, pues condiciona los patrones de comportamiento personal e interacción con otros individuos y con el medio; además intervienen directamente en la salud y bienestar de una persona. Es también un reto para los epidemiólogos en los aspectos de medición, análisis y modelaje. $^{20}$

Existen diversos instrumentos para determinar la función cognitiva y el nivel de depresión. Por lo mismo, es importante aclarar que la selección del Minimental y la Escala de Depresión Geriátrica obedeció a las propieda des psicométricas, ya determinadas en varios estudios. ${ }^{12}$ Al comparar el nivel de deterioro mental (37.96\%) y el de depresión leve (40\%) y severa (15\%) de este trabajo, con un estudio de 65 personas mayores, de una comunidad en Porto Alegre, ${ }^{4}$ que detectó déficits cognitivos en el $29 \%$ y síntomas depresivos del 30\%, se perciben prevalencias superiores, probablemente explicadas por la condición de institucionalización de nuestra población. 
La valoración oral en esta investigación encontró que en las personas mayores estudiadas, el $88.4 \%$ tiene un COP-D de 28 , con un promedio de 27 y un rango entre 8 y 28 . Al comparar estos resultados con el ENSAB-III, se encuentran algunas diferencias, pues este trabajo se diseñó específicamente para personas mayores de 60 años institucionalizados y el ENSAB maneja en un solo grupo los mayores de 55 años provenientes de la población gene$\mathrm{ral}^{3}$.; sin embargo, establece con claridad un promedio de 19.7 y un aumento del 100\% en el número de dientes perdidos por caries, comparado con el grupo de 35 a 40 años.

El trabajo de la FOUN ${ }^{21}$ muestra un incremento del COPD, con un promedio de 27 comparado con el ENSAB III, también a expensas de un incremento en el porcentaje de perdidos de 66.5\%. Datos similares muestra Matíz, ${ }^{22}$ quien señala una cifra aún mayor para el grupo de gerontes institucionalizados.

El efecto del deterioro mental y del nivel de depresión sobre el COP-D, no se encontró estadísticamente significativo en este estudio.

La caries radicular es otro indicador importante de la salud oral en este grupo poblacional, a consecuencia de la reabsorción gingival, pues las superficies radiculares quedan expuestas al medio ambiente cariogénico de la cavidad oral; a pesar de esto, son pocos los estudios que han determinado su prevalencia a nivel poblacional. ${ }^{9,23}$

A nivel nacional tan sólo se conocen los datos del ENSABIII y de Matíz. El primero no lo determina como un indicador muy relevante para este grupo poblacional; el segundo muestra tendencia al aumento con la edad. 3,22

En concordancia, con lo estudios de Wallace ${ }^{10} \mathrm{y}$ Gustavsen ${ }^{23}$ el género masculino y el grupo etáreo de 65 a 79 años muestran las mayores prevalencias de RCI. En nuestro estudio ser hombre mostró un OR de 2.02, IC 95\% (0.96-4.2); la edad no fue estadísticamente significativa, sin embargo, es importante considerar los límites superiores del IC 95\% (Tabla 4).

No se encontraron estudios que evaluaran la asociación entre el estado mental y el RCI. Nuestros hallazgos no los destacan como factores de riesgo. No obstante, se debe mencionar el efecto de la higiene oral mediante el ICP, para la caries radicular, pues se detectó como un factor de riesgo evidente y significativo para RCI mayores 0 iguales a 20 (Tabla 4).
La enfermedad periodontal es uno de los principales problemas de salud oral en las personas mayores. El ENSABIII $^{3}$ muestra para el grupo de 60 y más años una prevalencia de cálculos entre 50 y 53\% aproximadamente, bolsas de 4 a $5 \mathrm{~mm}$ cercanas al 35\% y bolsas de $6 \mathrm{~mm}$ y más casi en el 10\%. El estudio de la FOUN, ${ }^{21}$ muestra cifras diferentes: gingivitis, 54.9\%; periodontitis leve, 14.1\%; moderada $5.9 \%$, y severa $1.9 \%$, probablemente debido a las características de la muestra, pues no tiene una base poblacional y además es una población preocupada por su salud oral, atendida en forma regular en la Facultad de Odontología.

En nuestro estudio el estado periodontal es aún más severo, probablemente debido a la condición de estar institucionalizados, como ya ha sido demostrado en otros estudios. ${ }^{24-27}$

La asociación entre el género masculino y la severidad de la enfermedad periodontal, detectada en este estudio, concuerda con un estudio realizado en Finlandia. ${ }^{28}$

La escolaridad se muestra como un factor protector de la condición periodontal, a pesar de no ser significativo en este estudio (Tabla 5).

El deterioro mental y el nivel de depresión son factores de riesgo importantes para la condición periodontal, pues tanto en el análisis bivariado como en el múltiple, se destacan con OR significativos a un nivel de probabilidad de 0.10 (Tabla 5).

Es importante considerar el estado de higiene oral, medido con el ICP, tanto los factores de riesgo que lo condicionan en las categorías de regular y mala, como su implicación en el riesgo para enfermedad periodontal (Tablas 2 y 5).

Los hallazgos de factores de riesgo como género masculino, deterioro mental y nivel de depresión (Tabla 2), sugieren un patrón similar a los ya descritos en otros estudios ${ }^{24,28}$ y plantean la necesidad de chequeos permanentes, remoción profesional de placa y asistencia en la higiene oral de la persona mayor institucionalizada.

La tendencia al mejoramiento de la higiene oral con el incremento de la escolaridad también fue demostrada en este estudio. 
El edentulismo total fue ligeramente superior al parcial. Su factor de riesgo más relevante fue la edad (Tabla 6). Hallazgos similares podrían derivarse de las prevalencias de necesidad de prótesis por grupo etáreo para los dos maxilares en el ENSAB-III. ${ }^{3}$

Estos hallazgos no concuerdan con el estudio de la FOUN, ${ }^{21}$ probablemente por las razones ya expuestas.

En concordancia con la literatura revisada la edad es un claro factor de riesgo para el edentulismo parcial o total en ambos maxilares. ${ }^{29.30}$

En cuanto al efecto del género no concuerdan los resultados de este estudio con el de Hand, ${ }^{30}$ pues encuentra prevalencias iguales en los dos géneros en todos los grupos etáreos y mayores incidencias en el género masculino, lo cual se explica probablemente por una mayor preocupación de las mujeres por su salud oral, que conduce a tratamientos restaurativos en lugar de la extracción.

Es importante considerar la pérdida dental en la persona mayor, pues estudios como el de Shimazaki, ${ }^{31}$ enfatizan que la pérdida de muchos dientes reduce las fuerzas oclusales especialmente en individuos edéntulos. Al disminuir la función oclusal hay problemas de deglución, masticación y selección de alimentos, lo que provoca un deterioro nutricional que aumenta la morbilidad y mortalidad en edéntulos y personas sin dentadura.

Entre los factores relevantes para la salud oral de la persona mayor están su condición mental y su percepción personal en cuanto a la necesidad de prótesis, ya que puede estar sobre-estimada por el odontólogo, además de otras consideraciones de carácter médico y farmacológico antes de planear una intervención para solucionar el problema de edentulismo. ${ }^{31-33}$

Igualmente es indispensable una relación positiva entre el paciente y el odontólogo, ya que ésta contribuirá al éxito del tratamiento dental, pues se requiere participación y cooperación permanente. ${ }^{34}$

De acuerdo con Per Axelsson, las estrategias de autocuidado son fundamentales en el proceso de obtener y mantener la salud oral; sin embargo, esto podría verse comprometido si existen condiciones mentales o fisicas deterioradas. $^{35}$
Llaman la atención los resultados de riesgo de los antecedentes médicos, ya que los evaluados (enfermedades cardiovasculares, tratamiento médico actual, consumo de medicamentos de control), se comportan como protectores, lo cual puede explicarse posiblemente por la atención médica adicional y regular que demanda un individuo institucionalizado con las características mencionadas.

Los procesos patológicos orales en el anciano se pueden considerar como parte de la degeneración general de los tejidos del sistema estomatognático, conformes con el proceso de envejecimiento. Sin embargo, es dificil establecer un patrón que separe lo patológico del proceso normal, aspecto que debe investigarse más a fondo, específicamente el relacionado con los cambios morfológicos y morfopatológicos, que se pueden producir con la edad, tomando en consideración los factores externos, como traumatismos, infecciones, degeneraciones, así como la intervención de factores psicológicos, sociales y culturales, para establecer una diferencia e incluso, conocer el origen o los mecanismos, que actúan tras un diagnóstico.

Los hallazgos de este trabajo plantean la necesidad de continuar evaluando la salud oral de la persona mayor en una forma integral; es decir, determinar cómo los aspectos sociales, culturales, funcionales, sensoriales, mentales y ambientales, la condicionan y cómo su alteración puede, a su vez, reflejar problemas generales de salud, y en especial, el aspecto nutricional y la interrelación social y afectiva con otros individuos.

Esta conclusión se apoya adicionalmente en el artículo de Mecklenburg, ${ }^{6}$ que establece como objetivo primordial, la necesidad de trabajar colectivamente para educar, afectar las actitudes e influenciar acciones consecuentes, que promuevan la salud oral de esta población y la adopción de indicadores adecuados en un nivel comunitario que permitan su evaluación.

La discapacidad mental en la persona mayor puede o no afectar su nivel de competencia y su capacidad para participar en las decisiones terapéuticas. Representa, además, un reto para el profesional de la salud y le plantea una serie de implicaciones éticas y legales, que ameritan un estudio serio y permanente. ${ }^{36-38}$ 


\section{BIBLIOGRAFÍA}

1. Naciones Unidas. Estrategia Internacional para La Acción 2002 sobre el envejecimiento. Comisión para el desarrollo social, en calidad de Comité de Preparación de la Segunda Asamblea Mundial sobre Envejecimiento. Reanudación de la primera sesión. Nueva Cork, 10-14 de Diciembre de 2001. (Adelanto de la Versión no publicada).

2. Curcio CL, Gómez JF. Capacidad funcional en ancianos. Rev Asoc Colomb Geront Geriatr 1998; 12: 25 - 33.

3. Ministerio de Salud. Estudio Nacional de Salud Bucal-ENSAB III. Bogotá, Colombia. Tomo VIII, 1999. p. 109 - 199.

4. Silberman C, Souza C, Wilhems F, Kipper L, Wu V, Diogo C, et.al. Cognitive deficit and depressive symptoms in a community group of elderly people: a preliminary study. Rev Saude Pública 1995; 29:444-50.

5. Reyes CA. Envejecimiento Normal. Rev Estom 1993; 3:12 -15.

6. Mecklenburg RE, Martin FR. Oral health objectives for the elderly in the year 2000. Gerodontics 1986; 2: 161- 163.

7. Kelsey JL, Whittemore AS, Evans AS, Thompson WD. Methods in observational Epidemiology. Second Edition.Oxford University Press, New York, 1996.

8. Dean AG, Dean JA, Coulombier D, Brendel KA, Smith DC, Burton AH, et.al. Epi Info, Versión 6: a word processing, database, and statistics program for epidemiology on microcomputers. Center for Disease Control and prevention, Atlanta, Georgia, U.S.A., 1994.

9. Pieschacón MP. Programa piloto de odontología preventiva con escolares del municipio de Bucaramanga. Bucaramanga, Colombia: (1998). Tesis de Grado.

10. Wallace MC, Retief DH, Bradley EL. Prevalence of root caries in a population of older adults. Gerodontics 1988; 4: 84 - 89.

11. Ainamo. Índice de necesidades de tratamiento periodontal comunitario. Gerencia de Salud. Proceso Promoción Prevención. Santa fe de Bogotá,1997.

12. McDowell I, Newell C. Measuring Health. A guide to rating scales and questionnaires. Oxford University Press, New York, 1996.

13. Pagano M, Gauvreau K. Principles of biostatistics. Duxbury Press, Belmont Ca, 1993.

14. Hosmer DW, Lemeshow S. Applied Logistic Regression. Wiley \& Sons, New York, 1989.

15. Hosmer DW, Taber S, Lemeshow S. The importance of assessing the fit of logistic regression models: A case study. Am J Public Health 1991; 8: 1630 - 1635.

16. Microsoft Excell, 5.0, Microsoft Corporation, 1997.

17. StataCorp. 1999. Stata Statistical Software: Release 6.0. College Station, TX: Stata Corporation.
18. Lynch M. Manual Práctico de Medicina Bucal. Tomo III. México: Interamericana, 1990.

19. Marín DJ. Es necesaria la Odontogeriatría?. Revista de la Federación Odontológica Colombiana. Salud para la tercera edad 2000; 59: 39- 44.

20. Morris MC, Evans DA, Hebert LE, Bienias JL. Methodological issues in the study of cognitive decline. Am J Epidemiol 1999; 149:789-93.

21. Munevar AM, Rojas JK, Marín DJ. Perfil epidemiológico bucal de los pacientes de 55 años y más que asisten a las clínicas del geronte a la Facultad de Odontología de la U.N. durante el primer semestre del año 2000 y cual es la percepción que tienen estos pacientes sobre su propio estado de salud bucal. Revista de la Federación Odontológica Colombiana 2002; 61:7 - 32.

22. Matíz J. Morbilidad oral en la población geriátrica. Oral Día 1992; $5: 41-49$.

23. Gustavsen F, Clive JM, Tveit AB. Root caries prevalence in a Norwegian adult dental patient population. Gerodontics 1988; 4:219223.

24. Pajukoski H, Meurman JH, Snellman-Grohn S, Sulkava R. Salud oral en pacientes ancianos hospitalizados y no hospitalizados habitantes de una comunidad. Oral Surg Oral Med Oral Pathol Oral Radiol Endod 1999; 88: 437 - 443.

25. Vigild M. Oral hygiene and periodontal conditions among 201 dentate institutionalized elderly. Gerodontics 1988; 4: 140 - 145.

26. Pereira AC, Castellanos RA, da Silva SR, Watanabe MG, Queluz DP, Meneghim MC. Oral health and periodontal status in Brazilian elderly. Braz Dent J 1996; 7: 97- 102.

27. Meneghim M, Pereira AC, Silva FR. Prevalencia de cárie radicular e condicho periodontal em uma populacho idosa institucionalizada de Piraciba SP. Pesqui Odontol Bras 2002; 16: 50 - 56.

28. Lappalainen R, Wildstrom E, Markkanen H. Periodontal condition, remaining teeth and dental health habits of aged in Finland. Gerodontics 1988; 4: 277 - 279.

29. Angelillo IF, Saglioco G, Hendricks SJH, Villari P. Tooth loss and dental caries in institutionalized elderly in Italy. Community Dental Oral Epidemiology 1990; 18:216 - 218.

30. Hand JS, Kohout FJ, Cunningham MA. Incidence of edentulism in a non-institutionalized elderly population. Gerodontics 1988; 4:13 - 17.

31. Shimazaki Y, Soh I, Saito T, Yamashita Y, Koga T, Miyazaki H, Takehara T. Influence of dentition status on physical disability, mental impairment, and mortality in institutionalized elderly people. J Den Res 2001; 80:340-345.

32. Nikoukari H, Ponichtera AJ, Potter D. Complete dentures: Perceived need vs. Professional evaluation ofan institutional geriatric population. Gerodontics 1988;4: 305 -309.

33. Berkey DB. Restorative dental considerations for the elderly. Gerodontics 1988; 4:340- 342. 
34. Gordon SR, Sullivan TM. Dental treatment planning for compromised or elderly patients. Gerodontics 1986; 2: 217- 222.

35. Axelson P. An introduction to risk prediction and preventive dentistry. Quintessence Publishing, Illinois, USA, 1999.

36. Bridgman AM, Wilson MA. The treatment of adult patients with mental disability. Part 1: Consent and Duty. Br Dental J 2000; 189:66-68.

37. Bridgman AM, Wilson MA. The treatment of adult patients with mental disability. Part 2: Assessment of competence. Br Dental J 2000; 189:143-146.

38. Bridgman AM, Wilson MA. The treatment of adult patients with mental disability. Part 3: The use of restraint. Br Dental J 2000; 189:195 $-198$. 